\title{
POLÍTICAS PÚBLICAS PARA EL TERCER SECTOR EN PUERTO RICO: LA EXPERIENCIA DE LA COMISIÓN ESPECIAL SOBRE EL TERCER SECTOR DEL SENADO DE PUERTO RICO, 2000-2004
}

\section{Migdalia Camacho Hernández ${ }^{1}$}

\section{Resumen}

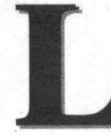

as políticas neoliberales que fundamentan la retractación del Estado como garantizador de derechos ciudadanos universales y del ofrecimiento de servicios de bienestar a la ciudadanía, ha sido un detonante en la aparición y fortalecimiento de las organizaciones del Tercer Sector tanto en Puerto Rico, como internacionalmente. En este trabajo reviso el Tercer Sector como concepto, como universo asociativo y en cuanto a su presencia en el contexto puertorriqueño. Planteo la importancia de desarrollar políticas públicas para este sector en nuestro país. Además, analizo las aportaciones de la Comisión Especial Sobre el Tercer Sector de la Legislatura de Puerto Rico 2000-2004, desde algunas dimensiones del concepto de estructura de oportunidades políticas y de la participación de las organizaciones en los procesos de desarrollar políticas públicas para el Tercer Sector.

Descriptores: políticas públicas, tercer sector, Comisión Especial sobre el Tercer Sector, participación del sector no lucrativo en política pública, participación en política pública.

\footnotetext{
${ }^{1}$ Catedrática Auxiliar en la Universidad del Turabo, Puerto Rico.
} 


\begin{abstract}
Neoliberal policies that justify the shrinking of the State as the overseer of universal rights and supplier of welfare services to its citizens, have triggered the rise and strengthening of the Third Sector in Puerto Rico and internationally. This paper reviews the Third Sector as a concept, as a linking universe, and as an important presence in the Puerto Rican context. I raise the importance of public policies development of this sector in our country. I also analyze the contributions by the Special Commission on the Third Sector of the Puerto Rico Legislature between 2000 and 2004 from some dimentions of the political opportunities structure' concept and of participation of organizations in the process of developing public policies for the Third Sector.
\end{abstract}

Key Words: public plicies, third sector; Special Commission on the Third Sector, non profit sector participation in public policy, public policy participation.

\title{
Introducción
}

Abordar las políticas sociales y los procesos que se generan en su formulación, implica hacer una mirada crítica a la manera que se entrelazan intereses e ideologías, así como las interrelaciones complejas entre los diversos sectores involucrados. Es menester reconocer en dicho proceso la importancia de los actores sociales que sustentan su accionar desde su concepción de lo que es la búsqueda del mejoramiento de la calidad de vida de la gente, el fortalecimiento de la ciudadanía y de los derechos de las personas. En este caso, hablo específicamente del Estado y del llamado Tercer Sector. Abordar las políticas sociales también implica una mirada a cómo los efectos de las políticas neoliberales han llevado a una reconceptualización de los roles y aportes de estos actores sociales.

Las políticas sociales en Puerto Rico se desarrollan dentro del contexto del Estado Benefactor de los Estados Unidos, modelo surgido en las primeras décadas del siglo 20, para enfrentar las crisis económicas (Colón, 2005; Lara, 2005). Según Colón (2005), el 
Estado Benefactor encontró sus límites a medida que la estructura económica cambió y su capacidad de controlar la política corporativa se deterioró. Según dicha autora, se hizo patente que el gasto público no podía seguir siendo el que estimulara el desarrollo económico y era necesario buscar otras estrategias para manejar la crisis. En ese contexto ideológico neoliberal, surgen como respuesta los procesos de reajuste estructural, que incluyeron propuestas de descentralización, privatización y focalización en las políticas sociales. Aunque vemos ya desde los años 60 intentos de reformular el Estado Benefactor, es en de la década de los 90 donde se articulan revisiones más amplias y más profundas. Todos los ajustes estructurales diseñados han implicado un cambio en la visión del rol del Estado en el manejo de las necesidades de la ciudadanía. Se vé al Estado, no como garantizador de servicios de bienestar, sino como facilitador en el mejor de los casos ó distanciándose del manejo de algunas de las necesidades y designándolas a otros actores sociales. De acuerdo con ésta versión, las políticas sociales no deben ser utilizadas con fines sociales (Rozas, 2005), son consideradas un 'gasto' (Guardiola, 1998) y no una inversión en el fortalecimiento de los derechos universales de la ciudadanía. Las políticas neoliberales se han implantado en muchas partes del mundo, a pesar de que estas tendencias han implicado un aumento en la desigualdad, la exclusión y la injusticia social. El aumento en los niveles de pobreza y la desigualdad social son las mayores manifestaciones de esta realidad (Colón, 2005; Lara, 2005; Kliksberg, 2003; Naciones Unidas, 2005).

En el caso de Puerto Rico, hay que analizar esta realidad reconociendo que la misma está impactada de manera significativa por sus relaciones políticas con los Estados Unidos. La inversión masiva de fondos federales en nuestro país determina en gran medida la manera en que se formulan y se implantan las políticas públicas en general. El impacto presupuestario de los fondos federales transferidos a Puerto Rico para el año fiscal 2006-07, se estimó en \$5,561.9 millones (Estado Libre Asociado de Puerto Rico, 2006). Para los próximos dos años (2009-2010) se espera además una inyección adicional de $\$ 5,000$ millones como parte de la Ley Federal de Estímulo Económico del nuevo Presidente de los Estados Unidos, Barack Obama. La posiblidad de diseñar e implantar políticas 
sociales que respondan a nuestras realidades, se ve amenazada fuertemente por esta realidad.

Guardiola (2002) entendió que la implantación de políticas sociales norteamericanas en el país se ha dado de manera indiscriminada y descontextualizada de nuestra realidad. La transferencia masiva de fondos federales produjo cambios significativos en el sistema de bienestar social del país (Pratts, 1987). Algunos de los cambios planteados por el autor fueron la pérdida de la capacidad de formular la política social, a la par que el sistema de bienestar social de Puerto Rico se convirtió en administrador de programas federales. Además, el gobierno federal intervenía directamente con la población al administrar programas sociales con un aumento significativo en la nómina del gobierno.

Se hace clara la necesidad de la participación en la vida pública de otros sectores sociales (Bertolotto, 2002), dada además la credibilidad limitada de las instituciones públicas en la protección de los derechos de la ciudadanía (Sanborn, 2002). La interacción compleja de los elementos planteados, entre otros, provocó una mayor escasez de alternativas en la satisfacción de necesidades de la ciudadanía. Esto permitió un aumento en el surgimiento de organizaciones de la comunidad o el fortalecimiento institucional de aquellas que existían previamente en el llamado Tercer Sector. En este trabajo abordaré algunos planteamientos conceptuales y operativos de este término y su pertinencia en Puerto Rico. También abordaré la participación del Tercer Sector en los procesos de desarrollo de políticas públicas. Finalmente analizaré el contexto de la Comisión Especial sobre el Tercer Sector del Senado de Puerto Rico 2000-2004 desde algunas dimensiones del concepto de "estructura de oportunidad política" (McAdam, McCarthy \& Zald, 2005), así como la participación de las organizaciones en influenciar las mismas.

\section{Tercer Sector}

El Tercer Sector como categoría conceptual

El fenómeno del Tercer Sector se ha hecho más fuerte a partir de la década del 80 , fortaleciéndose el desarrollo del mismo como uno 
diferenciado de los otros dos actores principales: el Estado y el Mercado. Siendo un fenómeno que se manifiesta de manera más dramática a partir de la década de los 80 's en el siglo 20 , la literatura sobre estos temas se focaliza mayormente en la diferenciación entre estos sectores, la controversia sobre la denominación y la conceptualización de este sector social, las teorías justificativas de su existencia en las economías y finalmente la valoración de su magnitud y su presencia social (Sajardo \& Chaves, 2006; Bertolotto, 2002).

El Tercer Sector como categoría conceptual está todavía en proceso de construcción y es ambigua (Olivera Rivera, 1998; Bertolo, 2003; Villar, 2001; Thompson, 1995). Es común que se utilicen para denominar este "universo asociativo", otros términos tales como: organizaciones comunitarias, organizaciones sin fines de lucro, organizaciones no gubernamentales (ONG'S), sector social, sector voluntario, sector independiente, organizaciones de la sociedad civil. Algunas denominaciones están dadas por marcos jurídicos, reglamentaciones, utilizados por el Estado, las propias organizaciones, organismos internacionales u otros (González \& Roitter, 2003). En general, se puede decir que el Tercer Sector está compuesto por diferentes organizaciones que no son capitalistas en cuanto que no buscan la acumulación de capital y cuyos excedentes no son distribuídos entre sus miembros. Tampoco están operadas por el Estado (Mertens, 2001; Roitter, 2004). De esta manera vemos un intento de precisar el concepto por lo que no es. Esto es visto como una limitación, así como también el que se trate como un sector a un universo asociativo altamente diverso en cuanto a objetivos, tamaño, lógicas de funcionamiento, prácticas sociales y otras características (Bombarolo, 2001; Estudios Técnicos, 2002; Roitter, 2004). Bombarolo (2001) considera que la heterogeneidad del sector es tan grande que parece "...casi imposible encontrar puntos vinculantes más allá del hecho de que se trata de organizaciones de personas". Otros, como Roitter (2004), entienden que utilizar el término 'sector'

\footnotetext{
${ }^{2} \mathrm{Al}$ utilizar el concepto de 'universo asociativo', me refiero a las organizaciones del Tercer Sector como un conjunto de organizaciones que comparten características y cumplen unas funciones y roles sociales determinados, los cuales se presentarán más adelante.
} 
permite estimar su importancia en la economía, sin implicar esto que se nieguen sus diferencias y contradicciones. Añade que las organizaciones pueden concebirse como tal ya que "forman parte de un universo que produce servicios o es expresión de intereses o proyectos públicos y que, a tal efecto, trabajan en un espacio diferenciable de la órbita estatal y de las empresas" (Roitter, 2004, p.21).

Por otro lado, Salamon y Anheier (1992) desarrollaron una definición estructural operativa utilizada frecuentemente. Esta definición caracterizó al Tercer Sector como uno que está compuesto por entidades que tienen una organización formal, son privadas, no lucrativas, autogobernadas, independientes del Estado y cuya membrecía es de carácter voluntario. Excluyó organizaciones como aquellas que son propiedad de trabajadores, que aunque generan lucro, tienen un fin social incuestionable.

Una de las áreas que causa mayor dificultad en el estudio del Tercer Sector es la que tiene relación con la clasificación de las organizaciones que pertenecen al mismo. Existen diversas clasificaciones y el asunto es de vital relevancia para poder estimar la presencia real del sector y su impacto en las economías nacionales. Salamon y Anheier (1996), diseñaron un sistema internacional para la clasificación de las organizaciones. El mismo fue organizado en diez categorías que a su vez se van dividiendo en otras subcategorías. Las categorías propuestas son: Cultura y Recreación, Educación e Investigación, Salud, Servicios Sociales, Ambiente, Desarrollo y Vivienda, Asistencia legal, Intercesión ("Advocacy") y Política, Intermediarios Filantrópicos, Actividades Internacionales y Religión. Estos desarrollos han permitido poder medir la presencia de este sector en diferentes partes del mundo y comparar los aportes del mismo en diferentes contextos nacionales (Salamon y Anheier, 1992 y 1996).

A las organizaciones del Tercer Sector se les atribuye en la literatura importantes atributos. Se entiende que estas organizaciones poseen suficiente flexibilidad como para poder reajustarse ante nuevos retos o condiciones en el contexto donde trabajan; promueven la 
participación ciudadana; son más pertinentes al trabajar cerca de la gente, por lo que pueden detectar más prontamente las necesidades de la ciudadanía; generan capital social, fortaleciendo los vínculos entre las personas y los demás elementos de su ambiente social; ayudan a colocar en la agenda pública nuevos asuntos que afectan la ciudadanía. Se les atribuye además valores basados en el altruismo, la solidaridad y el esfuerzo común. Se entiende que generan sentido de pertenencia y de dignidad en las personas.

Existen también fuertes críticas a lo que es el Tercer Sector y sus aportes a la vida pública (Villar, 2001; Roitter, 2004). Algunos consideran que las virtudes que se le asignan no están comprobadas. Thompson (1995) explicó cómo las ideas que se tienen sobre estas organizaciones producen que se tengan expectativas muy altas de las mismas como: que reduzcan el gasto público, produzcan servicios a más bajo costo, reduzcan las demandas gubernamentales, sean innovadoras en los servicios, satisfagan nuevas necesidades. Esto ha llevado a engrandecer las organizaciones como alternativa a la gestión pública, demonizando la presencia del Estado (Roitter, 2004). Es importante entonces poder reconocer las limitaciones del sector y así poder potenciar sus aportes a la sociedad donde se desenvuelve.

Petras (1999) planteó que las organizaciones no gubernamentales se han convertido en la cara comunitaria del neoliberalismo, despolitizando sectores de la población y cooptando los líderes potenciales en pequeños proyectos. Además, indica que el crecimiento de las ONGs coincide con el incremento en financiamiento bajo el neoliberalismo, que socavan el sentido de lo público en cuanto a la responsabilidad del Estado de velar por el bienestar de los ciudadanos; y que fomentan la idea neoliberal de que los problemas sociales son responsabilidad privada. Como consecuencia son los recursos privados los que tienen que atender éstos problemas. Es evidente, que existen fuertes puntos de tensión sobre la conceptualización de este sector de la sociedad y los roles ó funciones que cumplen en el desarrollo y consolidación de la democracia, lo que requiere mayor profundización. No obstante esto, existe una clara vinculación de las organizaciones con lo público y es importante comprender que la relación Tercer Sector-Estado es 
producto del momento histórico, cultural y político que lo contextualizan (Thompson, 2005; Villar, 2001; Bertolotto, 2002).

\section{Tercer Sector en Puerto Rico}

En Puerto Rico, al igual que en otras partes del mundo, el Tercer Sector, ha adquirido mayor visibilidad. Cada vez más, los sectores sociales le reconocen su rol en el desarrollo social y económico del país. A tales efectos, se está fortaleciendo la visión de éste como una alternativa a la gestión gubernamental en el ofrecimiento de servicios para atender las necesidades de la ciudadanía.

Bonamusa y Villar (1998) argumentan que el interés creciente por el estudio del Tercer Sector está relacionado "con la necesidad de encontrar una salida a la crisis del Estado de Bienestar y darle un nuevo papel a las organizaciones voluntarias". Evidencia de esto son las decisiones tomadas por el Gobierno de Puerto Rico, de establecer como una política pública del Estado, integrar las organizaciones del Tercer Sector al manejo de la deserción escolar (Hernández, 2005) y de los servicios a niños y niñas con necesidades especiales. También se han integrado las organizaciones sin fines de lucro a la gestión gubernamental en el desarrollo de campañas de prevención multisectoriales y otras iniciativas. Estas iniciativas reflejan una revalorización y reconocimiento creciente del Tercer Sector y de las posibilidades de alianzas entre éste y el sector gubernamental.

El uso de Tercer Sector como manera de denominar las organizaciones sin fines de lucro es relativamente nuevo en nuestro país; ya que la denominación generalizada ha sido la de 'sector sin fines de lucro'. Este sector ha cobrado una presencia que todavía no se conoce a cabalidad ni se le ha dado la importancia que requiere. Estudios Técnicos (2007) identificó unas 6,378 organizaciones sin fines de lucro operando en Puerto Rico. Esta investigación reveló que el sector tuvo un impacto significativo en la economía de Puerto Rico: aportó aproximadamente un 5.35\% al Producto Nacional Bruto (cerca de 3.042 millones), generó cerca de 229,608 empleos directos e integró servicios voluntarios que equivaldrían a unos 36,224 empleos a tiempo completo (este dato se duplicó desde el estudio que realizaron en el 2002). 
Los datos obtenidos por Estudios Técnicos (2007), además revelaron que las organizaciones ofrecieron servicios directos a alrededor de 800,00 personas. Se concluyó que de estas organizaciones no existir o de reducir sus servicios, se reportaría un aumento significativo en la carga fiscal del gobierno. En la encuesta que se llevó a cabo para dicho estudio, las organizaciones indicaron que ofrecían principalmente servicios sociales, seguido de servicios en educación y en tercer lugar servicios de salud. Este orden en la distribución confirmó las encontradas previamente en el estudio de necesidades de las OSFL, realizado por el Non-Profit Evaluation \& Resource Center, Inc. (2004). La investigación de Estudios Técnicos del 1996, también demostró una clara dependencia de las organizaciones del financiamiento gubernamental. $\mathrm{La}$ dependencia de los fondos gubernamentales tiene como consecuencia la gubernamentalización de las OSFL, afectan su participación en política pública y crean unas relaciones de poder desiguales entre dichos actores sociales (Camacho-Hernández, 2008).

Las organizaciones del Tercer Sector han sido instrumentales en la prestación de servicios a la ciudadanía en Puerto Rico, sea de una manera suplementaria o complementaria al Estado, además de establecer relaciones de colaboración con otras organizaciones y otros sectores de nuestra sociedad tales como empresas, entidades políticas y sindicatos. Algunas dimensiones de colaboración fueron identificadas en estudios del Tercer Sector (Estudios Técnicos, 2002 y 2007). La relación con las organizaciones también le ha permitido al Estado visualizar necesidades emergentes y aquellas que están pobremente servidas.

\section{Tercer Sector y Políticas Públicas en Puerto Rico}

Algunos actores de organizaciones sin fines de lucro consideran que las organizaciones del Tercer Sector deben actuar al margen de la política, por miedo a las consecuencias que esto puede resultar para las mismas (Camacho-Hernández, 2008). No obstante, la historia demuestra que entidades comunitarias han estado ligadas a la formulación y transformación de políticas del Estado, así como a los arreglos institucionales inherentes a estos cambios y a la relación directa con los diversos actores que intervienen en esos procesos. 
Existe una larga historia en el país de luchas comunitarias, ambientales, en defensa de grupos excluídos, derechos básicos y otras, que han hecho visibles las interacciones de organizaciones del sector con las políticas estatales. Algunos ejemplos lo son el caso de la lucha del pueblo puertorriqueño para sacar la Marina de Guerra de los Estados Unidos de la isla municipio de Vieques, la labor realizada por Casa Pueblo, la Alianza Comunitaria, Caborrojeños Pro Salud y Ambiente. Han incluído también luchas generadas o desarrolladas con la participación de múltiples organizaciones del Tercer Sector entre otros asuntos tales como: la contaminación del aire en Cataño, la protección de los derechos de las personas con impedimento y pacientes de VIH, entre tantos esfuerzos. Estas aportaciones, proyectan una función importante en cuanto a la participación ciudadana en los asuntos en la agenda pública y por ende en el fortalecimiento de la democracia; y han contribuido al desarrollo de una cultura de solidaridad entre actores sociales en la lucha por los derechos ciudadanos.

A pesar de estas aportaciones, muchas organizaciones están ensimismadas en su labor cotidiana y se limitan a su contexto local. Entiendo que esto es parte de lo que Lara (2005) denomina la 'mentalidad de kiosko', que no permite ver la totalidad de los contextos y la necesidad de una mayor articulación entre los diferentes actores sociales. Esto puede proyectar una imagen del sector como fragmentado y con poca participación en procesos de política pública. Sobre este particular, Fonseca Lago (2008) encontró una falta de articulación en las redes de organizaciones que estudió; y que las mismas estaban desconectadas entre sí aunque tenían metas comunes, y no tenían estrategias sistemáticas de colaboración entre ellas.

EstudiosTécnicos (2002) informó que el $52.4 \%$ de las organizaciones encuestadas desconocían si la legislación, política pública, reglamentos y ordenanzas que se habían emitido desde 1996 hasta el momento del estudio las habían impactado adversamente. En el estudio de 2007 , esto se redujo a un $25 \%$, reconociéndose un impacto negativo en un $60 \%$ de las OSFL encuestadas, del impuesto a las ventas (IVU). No obstante, sigue siendo preocupante la comprensión 
que estas organizaciones pueden tener sobre el impacto real de las decisiones gubernamentales en sus operaciones y de la relevancia de su participación en el desarrollo de políticas públicas. Esto debe ser un punto de reflexión para las organizaciones.

En la relación Estado-Tercer Sector ha permeado la desconfianza de las organizaciones hacia la convocatoria del gobierno y su temor a ser cooptadas por sus estrategias políticas. El desprestigio de representantes políticos, que se traduce en desconfianza del pueblo hacia las instituciones públicas y sus representantes, abona a la desconfianza y a la comprensión de las implicaciones que tiene el cruzar las políticas sociales y los intereses político partidistas. Este tema es una fuente de fuerte debate y ha generado cuestionamientos sobre posibles fracturas en la gobernabilidad en nuestro país. La visión negativa del Estado como uno altamente burocratizado dificulta la coordinación y cumplimiento de las expectativas del sector. Se plantea aquí una fuente de tensión que se relaciona con la necesidad del sector de tener autonomía social, operacional y política versus la necesidad de articular sus estrategias con el Estado. Estos elementos, entre otros, no facilitan esta relación, pero pueden convertirse en fuentes de oportunidad ante las realidades extremadamente complejas que se viven en el país y la necesidad imperiosa de convergencias sociales en pro del mejoramiento de la calidad de vida.

La participación de las organizaciones en la formulación de políticas públicas en Puerto Rico se da a través de un repertorio de estrategias para influenciarlas. Algunas de estas son: influenciar directamente los legisladores, asesores y otros actores en las diferentes fases en el proceso de definir la agenda de política pública; participar de grupos sectoriales donde se analizan ó concertan agendas de política pública tales como consejos, foros $\mathrm{u}$ otros; influenciar en el diseño de programas de partidos u otras entidades políticas; presentar casos a nivel judicial como alternativa para visibilizar dificultades relacionadas a derechos universales de la ciudadanía y para crear jurisprudencia en torno a los mismos; usar los medios de comunicación social para divulgar y hacer públicas sus agendas, entre otras. 
La participación en política pública del Tercer Sector en nuestro país puede ser abordado desde el concepto de 'estructura de oportunidades políticas'. Este es un concepto que se ha utilizado para explicar aspectos de la emergencia de movimientos sociales revolucionarios (McAdam, McCarthy \& Zald, 2005), y que se relaciona con el ambiente político que puede ser favorable ó no para el desarrollo de demandas y su articulación con los intereses de las élites. Estos autores enfatizan que entre la oportunidad, la organización y la acción colectiva median distintos factores, incluyendo los culturales, los cuales son interdependientes. No obstante, la estructuración de oportunidades políticas, es uno de central importancia. Aunque reconocen la falta de precisiónen del concepto, McAdam, McCarthy \& Zald (2005) le atribuyen tres dimensiones: la apertura o cierre relativo del sistema político institucionalizado; la estabilidad en los alineamientos de las élites que típicamente apoyan la política; la presencia de alianzas entre las élites; la capacidad y propensión del Estado a la represión.

En Puerto Rico, históricamente no ha habido una visión de desarrollar políticas públicas para el Tercer Sector. El énfasis en la relación con el Estado ha privilegiado la prestación de servicios, dentro de las líneas de políticas desarrolladas 'desde arriba'. No se había detectado un interés especial en fortalecer sus prácticas y su presencia social. Esto ha planteado una necesidad imperiosa de desarrollar políticas públicas en el país para un sector que promete seguir fortaleciéndose e impactando más ámbitos de nuestra realidad. Un esfuerzo hacia esa dirección surgió en el cuatrenio del 2000 al 2004, donde se creó en el Senado de Puerto Rico, la Comisión Especial Sobre el Tercer Sector. Esta experiencia la analizo a continuación como una oportunidad para que las OSFL se involucraran en el desarrollo de políticas públicas para su sector.

La Comisión Especial Sobre el Tercer Sector como una Estructura de Oportunidad Politica

La Comisión Especial sobre el Tercer Sector del Senado de Puerto (CETS) se creó en mediante la Resolución del Senado 741. Fue presidida por la ex senadora Margarita Ostolaza y creada a los fines de evaluar la condición de las organizaciones sin fines de lucro y su 
potencial de contribuir al desarrollo económico del país (Informe Final de Comisión, 2004). A través de dicha Comisión, se desarrollaron y se analizaron diversos proyectos, algunos de los cuales se convirtieron en leyes. El Informe Final sometido indicó que la Comisión produjo una "amplia cosecha de proyectos de ley", que la mayoría se convirtieron en leyes y que en general se cubrieron todos los ámbitos sugeridos por los hallazgos y conclusiones de la misma. Las necesidades de las organizaciones identificadas en general se relacionaron con aspectos de supervivencia económica (fomentar las donaciones y aportaciones filantrópicas); ausencia de un sistema jurídico claro que propiciara el desarrollo del sector sin fines de lucro y de una infraestructura que le preste servicios; serias complicaciones burocráticas; un mecanismo de auditabilidad que vele por la transparencia y buen funcionamiento administrativo. La Comisión informó que trabajó con todas, excepto atender su auditabilidad. Se produjeron veintidós (22) proyectos, de los cuales doce (12) fueron convertidos en Ley. El resto se quedó en alguno de los puntos del proceso de formulación de leyes en Puerto Rico. Se informó además que el estudio realizado tuvo el insumo de de representantes de 48 entidades que incluyeron organizaciones, empresas privadas y entidades públicas. También se refirieron a que entraron "en contacto" y tuvieron el "insumo y apoyo" de unas 74 organizaciones sin fines de lucro. Estos contactos fueron directos con las organizaciones o a través de las "gestiones relacionadas con la evaluación de solicitudes de fondos legislativos gestionados en la oficina de la Senadora Margarita Ostolaza” (pág. 4, énfasis de la autora).

A mi entender la CETS se convirtió en el primer esfuerzo firme y articulado de parte del Gobierno de Puerto Rico en darle atención al Tercer Sector e interesarse por conocer sus necesidades y realidades. La cosecha de legislación fue la mayor registrada hasta ahora para el sector y pretendió atender las preocupaciones del mismo. Se debe analizar la labor de esta Comisión en el contexto de una administración pública que intentó llegar a las comunidades pobres del país como una manera de adelantar agendas políticas, y reconocer que estas medidas son presentadas en un momento donde la crisis fiscal del gobierno comienza a presentar signos inequívocos de su 
presencia. No obstante, estos esfuerzos deben también verse desde la perspectiva de las oportunidades que les brindó a las organizaciones para visibilizarse y reclamar el espacio social que en justicia se han ganado.

A continuación utilizo algunas de las dimensiones planteados por McAdam, McCarthy \& Zald (2005) sobre las estructuras de oportunidades políticas para analizar la experiencia de la CETS. Dichas dimensiones de análisis pueden aplicarse o no en función de lo que se pretende analizar. Una perpectiva es que la CETS sirvió como una oportunidad para las organizaciones al representar una apertura en el proceso de formulación de política pública sobre el sector. Hasta la creación de la CETS, en Puerto Rico no había una política pública clara sobre el Tercer Sector, ni un reconocimiento oficial de su valor y de sus aportaciones al país. Visto de esa manera, la CETS se convirtió en una oportunidad para que el Tercer Sector afianzara su presencia y comenzara a articularse para lograr el mayor impacto posible en el proceso de influir en la agenda. Esto no parece haber ocurrido desde las organizaciones.

Las políticas públicas para el Tercer Sector construídas en el CETS, se montan desde un modelo de colaboración. No obstante, surge la preocupación de lo que parece ser una participación limitada de las organizaciones, considerando un universo de por lo menos 4,347 según el estimado disponible en el momento de surgir la Comisión (Estudios Técnicos, 2002). No se pudo conseguir información documentada en la Oficina de Servicios Legislativos de la Legislatura de Puerto Rico, más allá del informe final de la CETS, que fuera más precisa sobre cómo se hizo la convocatoria ni sobre los factores que pudieron influenciar en dicha participación.

No hay simetría en la participación de las organizaciones. Más bien existe una tendencia a la participación mayor de las fundaciones, organizaciones con más recursos y organizaciones del área metropolitana. En los informes relacionados a las medidas trabajadas por la CETS, se evidenció la pobre participación que organizaciones del Tercer Sector tuvieron en las vistas públicas convocadas. $\mathrm{La}$ medida que más participación directa tuvo fue la que se convertiría en 
la Ley 452 del año 2004, con la presentación de doce (12) organizaciones. Esta ley añadió un capítulo a la Ley de Corporaciones de Puerto Rico relacionado específicamente a las organizaciones sin fines de lucro. La segunda que más participación directa tùvo fue la que se convirtió en la Ley Núm 261 del año 2004, Ley del Voluntariado de Puerto Rico, con la participación de siete (7) organizaciones. Las recomendaciones de cambios en los proyectos fueron limitadas y superficiales en algunos casos. A mi juicio, la comparecencia de algunas agencias fue sólo para consignar su apoyo a las medidas.

La CETS realizó vistas públicas de contenido general, cuya documentación no fue localizada por la Oficina de Servicios Legislativos. Es posible que el patrón de participación de las organizaciones fuera diferente en esas experiencias, pero no hubo documentación disponible al momento de realizarse este trabajo, para determinarlo. Sería interesante conocer cómo se determinó la participación y se garantizó que hubiese representación de un sector que ya hemos descrito como uno muy diverso. Posiblemente el elemento diversidad al que aludí anteriormente, haya influenciado en que las organizaciones se sintieran convocadas.

Existe asimetría en la participación de las organizaciones en asuntos de política pública ya que las oportunidades no son homogéneas para el conjunto de las organizaciones (Bonamusa y Villar, 1998; Garay, 2001). Las organizaciones difieren además en los recursos con los que disponen, el nivel de influencia que creen tener y en los tipos de acercamientos que articulan con las instrumentalidades públicas. Estos elementos impactan la manera en que las organizaciones se visualizan a sí mismas en su relación con los círculos de poder y cómo interpretan su marco de oportunidades. Llama la particular atención los contactos que se tuvieron con las organizaciones desde la CETS, con las organizaciones. En primer lugar nos referimos a una participación generada y dirigida por el Estado ('arriba hacia abajo'), lo cual debe verse dentro del contexto de la historia de relaciones con elementos de desconfianza, analizados previamente. 
Podríamos concluir que esta participación fue una dirigida desde las élites políticas. Entiendo que esto por sí solo, puede verse como un elemento mediatizador de la naturaleza de la relación y la participación en el proceso que analizo. Finalmente, de 22 proyectos, un total de siete (7) medidas impactaban necesidades de organizaciones particulares como Fondos Unidos, la Fundación Comunitaria de Puerto Rico, las Corporaciones de Trabajadores Dueños, Museos y otras entidades. Esto es cónsono con el concepto de asimetría en la participación.

Otro elemento a ser analizado en esta dimension de estructura de oportunidad política, es el contexto legislativo en su dimension más amplia. Independientemente de la labor realizada por la CETS, los proyectos presentados tuvieron que pasar por las diferentes fases del proceso de desarrollar políticas en la Legislatura de Puerto Rico. Eso implica variedad de intereses, individuos, ideologías y agendas de las agencias gubernamentales y grupos de interés, que pueden viabilizar o no los proyectos. Se trata de procesos políticos muy complejos que hay que conocer y saber manejar, donde el entrejuego de estos elementos pueden ser no armonizables ó abiertamente conflictivos. Habría que incluir en este punto la inexistencia de mecanismos coordinados y articulados desde las mismas organizaciones, convocadas o no, para influir en la agenda legislativa para el Tercer Sector. Las medidas en general atendían preocupaciones de las organizaciones ó sectores de éstas. Las medidas que atendían asuntos sustantivos de necesidad del sector en general y que fueron convertidas en ley incluyeron: Ley 462 del año 2004 (crea un programa de internados de capacitación para las organizaciones sin fines de lucro); Ley 452 del año 2004 (añade un capítulo específico para las organizaciones sin fines de lucro en la Ley de Corporaciones de Puerto Rico); Ley 261 del año 2004 (Ley del Voluntariado de Puerto Rico).

Desde la segunda y tercera dimensión de la estructura de oportunidades, vemos alineación relativa en los planteamientos de las élites y entre ellas. Se consiguió un consenso entre los actores sociales que se involucraron en el proceso, en cuanto a reconocer la importancia del Tercer Sector en nuestra sociedad. El mismo estuvo 
abierto a recibir los insumos de élites políticas que son parte de la administración de turno y representantes de las élites económicas (bancos, asociaciones empresariales, farmacéuticas y otros). No obstante, este consenso no pareció ser el mismo en relación a la aprobación de proyectos que incluían determinaciones que impactaban el funcionamiento de alguna agencia o su presupuesto, al asignarle nuevas responsabilidades. En ese caso, los proyectos no fueron aprobados ó requirieron la negociación de enmiendas. Esto fue el caso con la Ley 452 del año 2004, en el que se negoció con el Departamento de Estado implantar por etapas una disposición de la ley.

Finalmente, es menester analizar qué fue lo que se quedó sin aprobar. Resulta interesante que dos de los aspectos más relevantes para las instituciones, relacionadas con la interación con la burocracia gubernamental y con el fortalecimiento económico de las organizaciones, fueron de poco logro real para la Comisión. Diferentes experiencias de interlocución entre organizaciones y gobierno, plantean que la interacción con la burocracia gubernamental es un factor limitante. Esto se observó en algunas ponencias de agencias en vistas públicas y en Congresos Nacionales de Política Pública para Asuntos Comunitarios, coordinados por la Red de Apoyo a Grupos Comunitarios en Desarrollo Socioeconómico, Inc., que se han celebrado desde el año 2000 en Puerto Rico. Las múltiples trabas en los procesos burocráticos de las agencias gubernamentales comienzan desde los procesos de someter las propuestas de proyectos, hasta los procesos de implantación y evaluación de los mismos. Las tardanzas en cada una de esas instancias, incluyendo los procesos de desembolsos de fondos, son la orden del día, con muy contadas excepciones. Esta realidad afecta de manera significativa el funcionamiento general de la organización, el clima organizacional y la prestación de los servicios; provocan malestar, frustración e inseguridad en todos los procesos, incluyendo los gerenciales. Esta situación fue denunciada por OSFL del sector salud una vez más recientemente, refiriéndose en esta ocasión a los fondos relacionados a VIH/SIDA (Parés, 2008); y surgió en otra investigación en Puerto Rico (Camacho-Hernández, 2008). 
El segundo aspecto, relacionado a la aprobación de medidas que ayudaran al sostenimiento económico de las organizaciones, fue otra área de poco logro. Aunque la CETS documentó la importancia de fomentar las donaciones filantrópicas, mejorar las exenciones contributivas y otras alternativas en esta dirección, la realidad fue que no se les dio paso a las mismas. En general, medidas importantes pero que impactaban el fisco, no fueron aprobadas. Ejemplos de lo anterior fueron los Proyectos del Senado 2433 (para conceder líneas de crédito a las organizaciones sin fines de lucro), el 2436 (lograr reducciones en canon de arrendamientos) y el 2543 (condonar deudas acumuladas por incumplimiento en la radicación de los informes anuales al Departamento de Estado). Esta legislación pudo darle alivios presupuestarios a las organizaciones, pero no fueron aprobados. Esto incluyó el Proyecto de la Cámara de Representantes 3234 que fue seguida de cerca por la CETS y que requería el pago del gobierno a sus suplidores de bienes y servicios, dentro de un período de treinta (30) días; la cual también se quedó fuera. Estas situaciones nos revelan que los procesos de desarrollar políticas públicas son impactados por diferentes variables, por lo que el éxito que se pueda tener en cumplir una agenda de política pública del Tercer Sector, requiere de mentes alertas, capacidad de negociar y detectar las oportunidades de influencias, así como la participación de todos los grupos interesados en las mismas.

\section{Implicaciones para la Administración Pública y el Tercer Sector}

La presencia del Tercer Sector en la sociedad puertorriqueña ha ido ganando un espacio en el ideario de los derechos y la satisfacción de las necesidades de la ciudadanía. Como toda representación social, enfrenta una multiplicidad de visiones y concepciones de lo que es y lo que representa en la sociedad. No obstante, más allá de esto, las realidades actuales que reforman el marco de responsabilidad del Estado en materia de garantizar derechos universales y la atención de las necesidades del pueblo, impone una mirada más profunda a estos actores sociales y a otros. También tiene implicaciones para la administración pública, que si sigue el modelo de colaboración implícito en las políticas públicas sobre el Tercer Sector que fueron aprobadas, está obligada a ampliar sus espacios de participación. 
Implica también una revisión de los procesos administrativos y los mecanismos para la toma de decisiones, los cuales deben ser cada vez más inclusivos y pluralistas. Esta realidad requiere seguir ampliando los espacios de participación real y efectiva de las organizaciones del Tercer Sector en todas las fases del proceso de desarrollar políticas públicas.

La lógica tradicional de un Estado que controla todo el proceso de desarrollar políticas públicas ('arriba hacia abajo'), tiene que ser revisado y reformulado. La situación actual que proyecta una crisis de legitimidad y confiabilidad en la toma de decisiones de los organismos estatales, requiere de mayores esfuerzos en la inclusión y la participación de los diversos actores sociales. Esta participación no puede ser circunstancial ni decorativa.

El cuadro crítico de la realidad puertorriqueña podría desembocar en un resquebrajamiento de la estabilidad del país y en los procesos de gobernabilidad democrática. Esto implica que no podemos desvalorizar ninguno de los mecanismos de respuesta con los que contamos. Se hace imperativa la necesidad de fortalecer todos los espacios, desde los familiares y comunitarios, la administración pública, las organizaciones del Tercer Sector y la sociedad civil en general, así como la responsabilidad social de las empresas. Es la interacción de esta multiplicidad de sectores la que conseguirá cambios reales, profundos y duraderos en esta realidad. Si diseñamos vías efectivas para la colaboración donde cada cual asuma su responsabilidad, podremos soñar con poder ver un mejor Puerto Rico. 


\section{Referencias}

Bertolotto, María I. (2002). Escenario de principios de siglo: Tercer sector y ONG. Boletín Electrónico Surá ,86.

Bombarolo, Félix. (2001). Mitos, misterios y epopeyas de las organizaciones sociales: la construcción cultural y el conflicto social no saben de sectores. Ponencia presentada en el III Encuentro de la Red Latinoamericana y el Caribe de la Sociedad Interamericana de Investigación del Tercer Sector (ISTR). Buenos Aires, Argentina.

Bonamusa, Margarita \& Villar, Rodrigo. (1998). Estrutura de oportunidades politicas y Advocacy: Elementos para un modelo político del Tercer Sector. Comunicacao apresentada no Primeiro Encontro da Rede de Pesquisas sobre o Terceiro Setor na América Latina e Caribe, do ISTR "Repensando o Público na América Latina: as organizacoes da sociedade civil". Río de Janeiro. Accesado el 12 de marzo de 2006 de www.rits.org.br/acervo-d/bonamusa.doc

Camacho-Hernández, Migdalia. (2008). La participación de las OSFL en la política pública de PuertoRico. Disertación doctoral inédita sometida a la Escuela Graduada de Trabajo Social de la Universidad de Puerto Rico, Río Piedras, Puerto Rico.

Cámara de Representantes de Puerto Rico. (2004). P. de la C. 3234.

Colón, Linda. (2005). Pobreza en Puerto Rico: Radiografia del proyecto americano. Puerto Rico: Luna Nueva.

Concepción, Carmen. (1999-2000). Justicia Ambiental, luchas comunitarias y Política pública. Revista de Administración Pública, 31 (32), 89-113. Accedido el 10 de marzo de 2009 de: http:/graduados.uprrp.edu/planificacion/trabajos_facultad/pdf/carmen concepcion/justicia_ambiental. 
Estado Libre Asociado de Puerto Rico. (2006) Presupuesto de Puerto Rico en el año fiscal 2006-2007. Accedido el 29 de marzo de 2007 de: www.presupuesto.gobierno.pr/ Tomo_I/Referencia/Economia.pdf

Estudios Técnicos. (2007). Las organizaciones sin fines de Lucro en 2007: Una fuerza económica. San Juan, PR: Fundación Carvajal, Fundación Flamboyán., Fundación Banco Popular, Miranda Foundation, Fundación Ferré Rangel, Museo de Arte de Puerto Rico, Fundación José J. Pierluisi.

Estudios Técnicos. (2002). Estudio de las organizaciones sin fines de lucro en Puerto Rico. San Juan, P.R: Fundación Angel Ramos, Fondos Unidos de Puerto Rico, Fundación Comunitaria de Puerto Rico y Fundación Chana Goldstein y Samuel Levis.

Estudios Técnicos. (1996). Organizaciones sin fines de lucro en Puerto Rico. San Juan, P.R: Fundación Angel Ramos, Fondos Unidos de Puerto Rico, Fundación Comunitaria de Puerto Rico y Fundación Carvajal.

Garay, Candelaria. (2001). Incidencia de ONGs en políticas públicas: Las ONGs como actores políticos en el campo de la infancia $y$, tratamiento y prevención de HIV-SIDA. Tercer Encuentro de la Red Latinoamericana y del Caribe de Investigación, de Sociedad Interamericana de Investigación del Tercer Sector (ISTR). Accesado el 8 de marzo de 2006, de www.icd.org.uy/mercosur/ponencias/Garay.PDF.

González, Inés. \& Roitter, Mario. (2003). Ideas sobre sociedad civil: Pasado y presente. Ponencia presentada en la IV Conferencia de la Red Latinoamericana y del Caribe de la Sociedad Interamericana de Investigación del Tercer Sector (ISTR). San José, Costa Rica. Accesado el 25 de febrero de 2006 de: www.lasociedadcivil.org/new_index.phtml?ac=ciberteca\& 
Guardiola, Dagmar. \& Serra, José. (2002). Politica social y trabajo social en Puerto Rico: Desafios y alternativas para el siglo XX. Puerto Rico: Publicaciones Puertorriqueñas.

Hernández, Yadira. (2005, 28 de octubre). Giro en la distribución de CASA. El Nuevo Día. Accesado el 20 de marzo de 2006 de http://www.adendi.com.

Kliksberg, Bernardo (2003). Hacia una nueva visión de la política social en América Latina: Desmontando mitos. Revista Venezolana de Gerencia, 8 (21). Accesado el 10 de marzo de 2006 de $99842003003000002 \& \operatorname{lng}=\mathrm{es} \& \mathrm{nrm}=$.iso.

Lara, Lourdes. (2006). Las organizaciones comunitarias y la formulación de política pública. En Nilsa M. Burgos Ortiz (Ed.), Politica social y trabajo social, Serie Atlantea 3 (129). Puerto Rico: Proyecto Atlantea.

Ley núm. 261. (2004, 8 de septiembre). San Juan, Puerto Rico: Estado Libre Asociado.

Ley núm. 452. (2004, 23 de septiembre). San Juan, Puerto Rico: Estado Libre Asociado.

Ley núm 462. (2004, 23 de septiembre). San Juan, Puerto Rico: Estado Libre Asociado.

McAdam, Doug, McCarhy, John \& Mayer, Zald. (2005). Opportunities, Mobilizing structures and Framing Processes: Toward a Synthetic, Comparative Perspective on Social Movements. En Doug McAdam, John McCarhy \& Zald Mayer (Eds.). Comparative Perspectives on Social Movements: Political Opportunities, Mobilizing Structures and Cultural Framings. Estados Unidos: Cambridge University Press. 
Mertens, Sybille. (2001). Organizaciónes sin fines de lucro y economía social: Dos concepciones del "tercer sector". Sociología del Trabajo 33, agosto-julio. Accesado el día 10 de marzo de 2006 de: www.lasociedadcivil.org/new_index.phtml? next $=165 \&$

Naciones Unidas (2005). Informe Sobre Desarrollo Humano 2005. Accesado el 20 de mayo de 2006 de: http://hdr.undp.org/reports/global/2005/espanol/

Olivera, Alberto. (1998). Problemas conceptuales en el estudio de las organizaciones civiles: De la sociedad civil al Tercer Sector. México: Instituto de investigaciones histórico sociales, Universidad Veracruzana.

Parés, María. (2008, 6 de mayo). Gobierno “obligará “el cierre de organizaciones de base. El Nuevo Día. Accedido el 20 de marzo de 2006, http://www.endi.com.

Pratts, Raúl (1987) La política social en Puerto Rico. Santurce, Puerto Rico: Ediciones Porta Coeli.

Petras, James. (1999). América Latina: De la Globalización a la Revolución. Argentina: Ediciones Homo Sapiens.

Rozas, Margarita. (2006). Democratización, Responsabilidad Social y Derechos sociales. En Burgos, N. (Ed.). Politica social y trabajo social, Serie Atlantea (49-63). Puerto Rico: Proyecto Atlantea, Universidad de Puerto Rico.

Roitter, Mario. (2004). El tercer sector como representación de la sociedad civil. En: Daniel Mato (Coord.) Politicas de ciudadanía y sociedad civil en los tiempos de la globalización (17-32). Caracas: FACES, Universidad Central de Venezuela.

Sajardo, Antonia \& Chaves, Rafael. (2006). Balance y tendencias en la investigación sobre el Tercer Sector no lucrativo. Revista de Economía Pública, Social y Cooperativa, 56, 87116. 
Salamon, Lester. \& Anheier, Helmut. (1996). The nonprofit sector: A new global force. Working papers of the Johns Hopkins comparative nonprofit sector project no.21. Johns Hopkins University. Accesado el 20 de febrero de 2006 de: www.jhu.edu/cnp/.

Salamon, Lester. \& Anheier, Helmut. (1996). The international classification of nonprofit organizations. Working papers of the. Johns Hopkins comparative nonprofit sector project ,no.19. Johns Hopkins University. Accesado el 20 de febrero de: 2006 de www.jhu.edu/ccss/pubs/pdf/pakistan.pdf.

Salamon, Lester. \& Anheier,Helmut. (1992). In search of the nonprofit sector II: The problem of classification. Working papers of the Johns Hopkins comparative nonprofit sector project, no.3. Accesado el 20 de febrero de 2006 de: www.jhu.edu/cnp/

Senado de Puerto Rico. (2004). Informe final de la comisión sobre el tercer sector. San Juan, Puerto Rico.

Senado de Puerto Rico. (2004). P.del S. 2433.

Senado de Puerto Rico. (2003). P. del S. 2543.

Thompson, Andrés. (1995). Políticas públicas y sociedad civil. Argentina: CEDES. Accesado el 1.0 de marzo de 2006 de: http://biblioteca virtual.classo.org.ar/ar/libros/argentina/cedes/thom4.rtf.

Villar, Rodrigo. (2001). El tercer sector, la sociedad civil y la gobernabilidad democrática en Colombia. En: Pensando el desarrollo rural desde la formación del capital social. Accesado el 10 de marzo de 2006 de: http://www.paisrural.org/memorias/rodrigo_villar.pdf.

Zayas, Herminia.(2006). Análisis de Veinte Años en la Lucha Ambiental de Puerto Rico del 1980 al 2000. Tesis de maestría no publicada, Universidad Interamericana, Ponce, Puerto Rico. 